\title{
An Intelligent Chatter Detection Method Based on EEMD and Feature Selection with Multi-channel Vibration Signals
}

Yun Chen ${ }^{1}$, Huaizhong $\mathrm{Li}^{2 *}$, Liang $\mathrm{Hou}^{1}$, Jun Wang ${ }^{3}$ and Xiangjian $\mathrm{Bu}^{1}$

${ }^{1}$ Department of Mechanical and Electrical Engineering, Xiamen University, Xiamen, China

${ }^{2}$ Griffith School of Engineering, Gold Coast campus, Griffith University, Australia

${ }^{3}$ School of Mechanical and Manufacturing Engineering, University of New South Wales, Sydney, Australia

\begin{abstract}
Chatter detection in metal machining is important to ensure good surface quality and avoid damage to the machine tool and workpiece. This paper presents an intelligent chatter detection method in a multi-channel monitoring system comprising vibration signals in three orthogonal directions. The method comprises three main steps: signal processing, feature extraction and selection, and classification. The ensemble empirical mode decomposition (EEMD) is used to decompose the raw signals into a set of intrinsic mode functions (IMFs) that represent different frequency bands. Features extracted from IMFs are ranked using the Fisher discriminant ratio (FDR) to identify the informative IMFs, and those features with higher FDRs are selected and presented to a support vector machine for classification. Single-channel strategies and multi-channel strategies are compared in low immersion milling of titanium alloy Ti6A14V. The results demonstrate that the
\end{abstract}

\footnotetext{
* Corresponding author: Dr Huaizhong Li, School of Engineering and Built Environment, Gold Coast campus, Griffith University, QLD 4222, Australia. E-mail: lihuaizhong@gmail.com; h.li@griffith.edu.au. Telephone: +61 (7) 5552 8252; Facsimile: +61 (7) 55528062
} 
two-channel $(A y, A z)$ strategies based on signal processing and feature ranking/selection give the best performance in classification of the stable and unstable tests.

Keywords: Chatter detection; ensemble empirical mode decomposition; Fisher discriminant ratio; support vector machine; multi-channel fusion; machining.

\section{Introduction}

Machining operations are enhanced due to the development of new concepts, devices, materials, tools and structures. However, machining stability is still a limitation which may cause poor surface quality, reduced tool life, and even breakage of cutting tools in metal removal processes [1]. One of the main problems with machining stability is chatter, which is a self-induced vibration between the cutting tool and workpiece with complex, nonlinear and nonstationary characteristics. In order to avoid chatter, extensive studies have been devoted to obtain the stability chart in the two-dimensional space of the spindle speed and depth of cut [2]. However, those studies require a complete analysis of the machining system dynamics and in-depth knowledge of the machining process and material properties, which is difficult for industrial users. On the other hand, intelligent monitoring is preferred in industrial applications, given the recent advances in sensors and signal processing techniques.

For an accurate diagnose of the machining process, monitoring systems rely on usage of sensors to detect tool or workpiece malfunctions. Dynamometers [3], accelerometers [4], microphones [5] and AE sensors [6] are commonly-used sensors to monitor the machining operations. Delio et al. [7] compared the three types of sensors for chatter detection and 
control. The results indicated that the force signals failed to adequately reflect chatter in low-immersion and low-feed machining operations due to the short contact time, whereas microphones were more suitable to chatter detection than other remotely placed displacement sensors. Nevertheless, microphones are limited by directional considerations, low frequency response and environmental sensitivity. The suppression of environmental noise is mandatory for chatter detection using microphones [8]. Kuljanic et al. [9] investigated multi-sensor approaches for chatter detection, and the results showed that a multi-sensor system composed of the axial force sensor and accelerometers gave the best results than any single-sensor or multi-sensor system in their investigated milling system.

Signal processing techniques extract from the signals the features related to chatter. Those features can be used as either chatter indicators or inputs to an intelligent system for chatter detection. Schmitz et al. [10] evaluated the statistical variance in the once-per-revolution sampled AE signal during milling as a chatter indicator. Vela-Martinez et al. [11] used a fractal rescaled range analysis method to evaluate signal fractality that is related to changes in machining stability. Patwari et al. [12] used the fast Fourier transform (FFT) to analyse chatter that is generated by chip serration in titanium machining. Alternatively, the time-frequency analysis techniques are used for feature extraction. The wavelet packet [13], Wigner time-frequency [14] and Hilbert-Huang transform [4] have been used to different machining systems for chatter detection. The time-frequency methods predominate over the time or frequency analysis methods, as they can reflect the nonstationary properties of signals over time. 
Chatter is related to the dynamic behavior of the machine-tool-workpiece system. There is a frequency-dependent energy rise around system natural frequencies when chatter occurs. The frequency bands that cover the natural frequencies are usually extracted from the raw signals, therefore highlighting the incipient characteristics and improving the sensitivity of the detection method. Lamraoui et al. [15] designed a multiband resonance filtering to process the signals before feature extraction. Yao et al. [16] applied a wavelet packet transform to decompose the raw signals and identify the most sensitive frequency bands related to chatter. From those components, statistical features, such as mean, variance, skew and kurtosis can be extracted to describe the raw signals. However, not all features can adequately reflect the changes in the machining states. Hence, a feature selection criterion or reduction method is needed [17]. Dong et al. [18] applied neural networks to select relevant features, and Lamraoui et al. [15] ranked features according to relative entropy measure. In-depth discussions about feature selection techniques can be found in the review papers $[17,19]$.

The role of pattern recognition methods employed in the implementation of intelligent monitoring systems is a fundamental one. A number of techniques have been used to detect chatter based on sensor signal features. Siddhpura and Paurobally [20] and Teti et al. [17] in their review papers stated that neural network and fuzzy logic techniques are widely used in tool condition monitoring. Other methods, e.g. Gaussian mixed model [21] and support vector machine [16], have also been employed to recognize the machining states. In some cases, a single sensory source for measuring a particular variable may not be able to meet all the required performance specifications. A solution to this problem is data 
fusion that combines multiple data sources so that the results may be better than those when these sources are used individually [17]. In order to develop an intelligent multi-sensor chatter detection system, Kuljanic et al. [8] used four decision-level fusion strategies including linear combination of sensor chatter indicators, neural network, fuzzy logic and statistical inference. The results indicated that the statistical inference strategy achieved the highest levels of accuracy among all the fusion strategies. Based on the combination of three types of sensors, Segreto et al. [6] obtained a high success rate in the assessment of tool wear in nickel alloy turning using a back-propagation neural network.

This paper presents a study on chatter detection in a multi-channel monitoring system. The ensemble empirical mode decomposition (EEMD), an improvement of the empirical mode decomposition (EMD), is employed to decompose the raw signals into a set of intrinsic mode functions (IMFs) with varied frequency bands. Features extracted from each IMF are ranked based on the Fisher discriminant ratio (FDR), and top ranked features are selected to a linear support vector machine (SVM) for classification. Single channel strategies and multi-channel strategies are compared by assessing the vibration signals from three sensory channels in low immersion end milling titanium alloy Ti6Al4V under various cutting conditions. The methodology, experimental setup and analysis results are presented in the following sections.

\section{Methodology}

Chatter is a nonlinear and nonstationary dynamic instability, whose energy at specific frequency ranges varies according to the dynamic characteristic of the machining system 
$[8,22]$. It is not practical to determine such dynamic characteristic in industrial conditions. In order to obtain a monitoring system independent from the system dynamics, signal processing techniques and intelligent classification methods are conceived. The work aims to develop an intelligent chatter detection method based on EEMD and feature selection in a multi-channel monitoring system. EEMD that is a self-adaptive analysis for nonlinear and nonstationary signals decomposes signals into IMFs, which limits chatter frequencies in narrow frequency bands and highlights chatter characteristics. Moreover, when analyzing intermittent and noisy signals produced in interrupted low immersion milling, EEMD generates more effective IMFs than EMD without mode mixing. In order to improve the classification accuracy and save computation time, features with high separability capabilities are selected from the informative IMFs for classification. The fusion techniques of multi-channel signals are investigated and utilized to further improve the classification accuracy. The procedures for the proposed approach are discussed in detail in the following subsections.

\subsection{Ensemble empirical mode decomposition}

The EMD adaptively decomposes a nonstationary signal to a set of IMFs that represents simple oscillatory modes as a counterpart to the harmonic functions. Using EMD, an arbitrary signal $s\left(t_{n}\right)$ can be decomposed into $I$ oscillatory modes and the residue $r_{i}$

$$
s\left(t_{n}\right)=\sum_{i=1}^{I} c_{i}\left(t_{n}\right)+r_{i}
$$

where $t_{n}$ is the $n^{\text {th }}$ discrete time and $c_{i}$ is the $i^{\text {th }}$ IMF. Due to the adaptive property of EMD, the oscillatory mode with the highest frequency band of the signal $s\left(t_{n}\right)$ is decomposed into the first IMF $c_{1}$, and subsequent IMFs with progressively lower frequency bands. 
In order to improve EMD, Huang and $\mathrm{Wu}$ [23] used white noise to homogenize the whole time-frequency space and enhance the projection of different components of the signal into proper IMFs. The procedures of EEMD are as follows:

i. Add a white noise series $w_{j}(j=1,2, \ldots, J)$ with a zero mean and constant amplitude to $s\left(t_{n}\right)$ in the $j^{\text {th }}$ trial.

$$
s_{j}\left(t_{n}\right)=s\left(t_{n}\right)+w_{j}
$$

ii. Decompose $s_{j}\left(t_{n}\right)$ using EMD to generate IMFs $c_{i j}\left(t_{n}\right)$.

iii. Given an ensemble number of $J$, the final $i^{\text {th }}$ IMF is the ensemble mean of corresponding IMFs of all the trials

$$
c_{i}\left(t_{n}\right)=\frac{1}{J} \sum_{j=1}^{J} c_{i j}\left(t_{n}\right)
$$

The ensemble number $J$ should be large enough to decrease or completely cancel the added white noise by the ensemble means. An ensemble number of a few hundred will usually lead to an accurate result [23]. Yeh et al. [24] suggested that the amplitude of white noise should not be more than $20 \%$ of the standard deviation of the signal.

\subsection{Feature extraction and selection}

In order to describe the signals, the statistical features that are correlated with the machining states are extracted from each IMF. Different methods have been applied for feature extraction in time, frequency, or in time-frequency domains. In this work, seven features are investigated for chatter detection: energy ratio, peak-to-peak value (P2P), 
standard deviation of P2P per revolution, root mean square (RMS), crest factor, skewness and kurtosis. Table 1 lists the features for each IMF.

It is noted that the sensitivities of different features to chatter vary. Those features that are the most significant and reliable can help design a classifier with good performance. For this reason, the feature selection procedure is necessary. The FDR is used to rank features, and helps to select the top ranked features for chatter detection. The FDR of an individual feature in the two-class problem is defined as

$$
S_{i m}=\frac{\left(\mu_{w_{1}, i m}-\mu_{w_{2}, i m}\right)^{2}}{\sigma_{w_{1}, i m}^{2}+\sigma_{w_{2}, \text { in }}^{2}}
$$

where $S_{i m}$ is the FDR for the $m^{\text {th }}(m=1,2, \ldots, 7)$ feature extracted from $c_{i}, \mu_{1, i m}$ and $\mu_{2, i m}$ are the means corresponding to the feature under investigation for the first and second classes, respectively, and $\sigma_{1, i m}^{2}$ and $\sigma_{2, i m}^{2}$ are the variances. The FDR quantifies the separability capabilities of individual features. A larger $S_{i m}$ indicates a high separability capability, and vice versa [25].

Table 1. Features for each IMF $c_{i}$.

\begin{tabular}{ll}
\hline Features & Equations \\
\hline Energy ratio & $f_{i 1}=\sum_{n=1}^{N} c_{i}^{2}\left(t_{n}\right) / \sum_{i=1}^{I} \sum_{n=1}^{N} c_{i}^{2}\left(t_{n}\right)$ \\
Peak-to-peak value (P2P) & $f_{i 2}=\max \left(c_{i}\right)-\min \left(c_{i}\right)$ \\
Standard deviation of P2P per revolution & $f_{i 3}=\left(\sum_{k=1}^{K}\left(\max \left(c_{i}^{k}\right)-\min \left(c_{i}^{k}\right)\right)^{2} /(K-1)\right)^{1 / 2} *$ \\
Root mean square & $f_{i 4}=\left(\sum_{n=1}^{N} c_{i}^{2}\left(t_{n}\right) / N\right)^{1 / 2}$ \\
Crest factor & $f_{i 5}=\max \left(c_{i}\right) / f_{i 4}$ \\
Skewness & $f_{i 6}=E\left(c_{i}-\bar{c}_{i}\right)^{3} / \sigma^{3 *}$ \\
Kurtosis & $f_{i 7}=E\left(c_{i}-\bar{c}_{i}\right)^{4} / \sigma^{4}$ \\
${ }^{*} c_{i}^{k}$ is the data extracted from $c_{i}(t)$ at the $k^{\text {th }}$ spindle revolution; \\
${ }^{* * *} \sigma$ is the standard deviation of $c_{i}(t)$.
\end{tabular}




\subsection{Pattern recognition}

The SVM is a supervised learning method suitable for classification of small sample sizes [26]. SVMs separate classes using the training data by fitting an optimal separating hyperplane in a feature space. The optimization problem being solved aims to maximize the margins between the optimal hyperplanes and the closest training data (i.e. support vectors). For non-separable two-class cases, a brief summary of SVM theory is given. For more information, refer to Refs. [26-28].

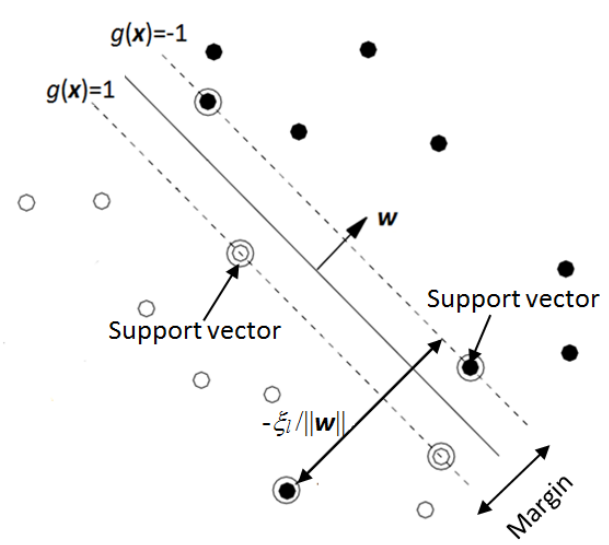

Fig. 1. SVM for the non-separable two-class case.

Let $\boldsymbol{x}_{l}, l=1,2, \ldots, L$, be the feature vectors of the training data set and $\boldsymbol{y} \in\{-1,1\}$ be the corresponding class labels. The decision function $g(\boldsymbol{x})$ is defined by the weight vector $\boldsymbol{w}$ and the threshold $w_{0}$ as

$$
\begin{aligned}
& g(\boldsymbol{x})=\boldsymbol{w} \cdot \boldsymbol{x}+w_{0} \\
& \text { If } \boldsymbol{w} \cdot \boldsymbol{x}_{l}+w_{0} \geq 1-\xi_{l} \quad\left(\leq-1+\xi_{l}\right), \boldsymbol{x}_{l} \in \text { Class } \boldsymbol{y}=1(-1)
\end{aligned}
$$

where the slack variable $\xi_{l}$ is non-negative. The support vectors lie on two hyperplanes $g(\boldsymbol{x})= \pm 1$, as shown in Fig. 1. The margin maximization between the two hyperplanes is solved using the following optimization problem 


$$
\operatorname{minimize} \frac{\|\boldsymbol{w}\|^{2}}{2}+C \sum_{l=1}^{L} \xi_{l}
$$

where $C$ is a positive constant that controls the relative influence of the two competing terms in Eq. (6). Using the Karush-Kuhn-Tucker conditions, Eq. (6) is modified as

$$
\operatorname{maximize}\left(\sum_{l=1}^{L} \alpha_{l}-\frac{1}{2} \sum_{l, m} \alpha_{l} \alpha_{m} y_{l} y_{m} \boldsymbol{x}_{l}^{T} \cdot \boldsymbol{x}_{\boldsymbol{m}}\right)
$$

where $\alpha$ with the subscript is the Lagrange multiplier and $\boldsymbol{x}_{l}^{T} \cdot \boldsymbol{x}_{\boldsymbol{m}}$ defines a linear kernel $[26,27]$. By replacing the linear kernel with nonlinear ones, Eq. (7) is extended for nonlinear SVMs that map the data into a higher dimensional Hilbert feature space. Such a mapping technique may result in an optimum hyperplane for a more complex class distribution, which may be not separable in the original feature space. The commonly used nonlinear kernels include polynomials, radial basis functions (RBF) and hyperbolic tangent [28].

\subsection{Detection strategies}

The detection strategies are summarized in Fig. 2. Firstly, each channel signal is processed using EEMD to obtain IMFs. Secondly, the features are extracted from each IMF and ranked by FDR. Thirdly, the top ranked features of each channel are inputted to a SVM classifier in order to implement a single-channel strategy. Finally, the hyperplane decision functions from single-channel strategies are processed using the multi-channel fusion strategies. The multi-channel strategies use an additional SVM [29] to fuse the decision functions from either two single-channel strategies or three single-channel strategies as the feature vectors to classify the signals. 


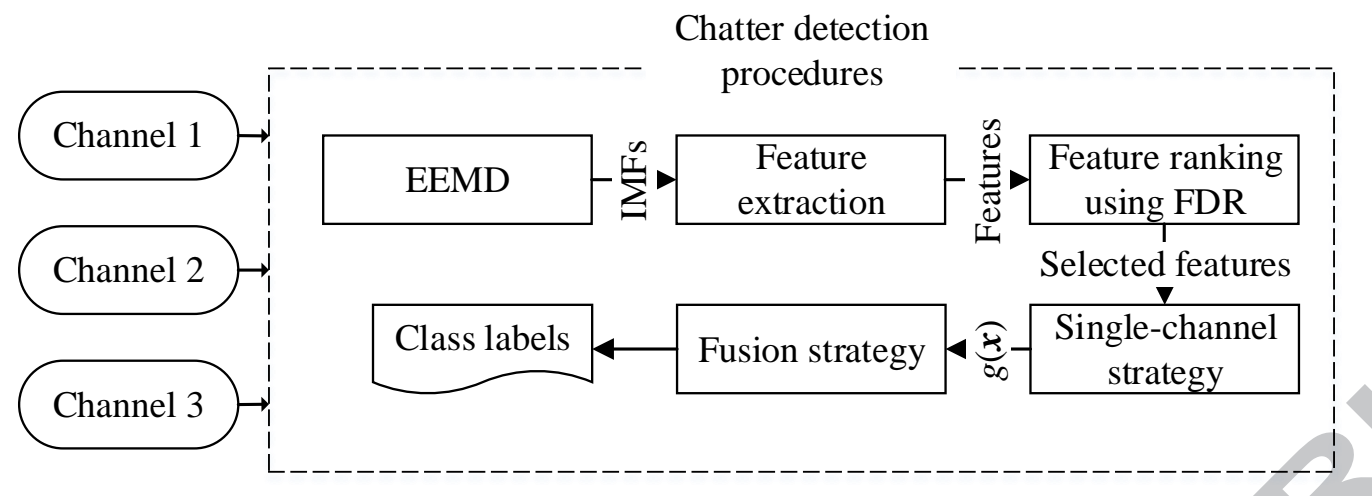

Fig. 2. Schematic diagram of the strategies for chatter detection.

\section{Experimental Assessment}

\subsection{Experimental setup}

The proposed approach for chatter detection was assessed with experimental data from end milling of titanium alloy Ti6Al4V. The milling tests were conducted on a Deckel Maho 5-axis CNC milling centre under dry cutting conditions. The experimental setup and the coordinate system of the vibration signals are shown in Fig. 3, in which the feed direction is along the $x$ direction. A PCB 356A16 accelerometer with three channels $A x, A y$ and $A z$ was mounted on the fixture to measure the vibration signals in the $x, y$ and $z$ directions, respectively. The sample rate for each channel was $20 \mathrm{kHz}$. Shoulder milling was performed throughout the tests. The cutter used was a Sutton E5060800 two-flute end mill with a diameter of $8 \mathrm{~mm}$ and a helix angle of $30^{\circ}$. A fixed overhanging length of $38 \mathrm{~mm}$ was utilised for the end mill. 


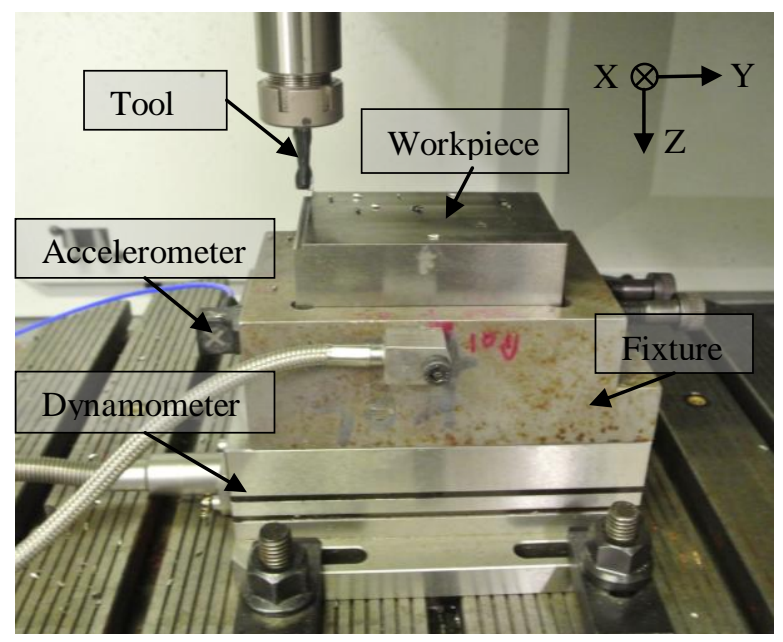

Fig. 3. Experimental setup for end milling of titanium alloy Ti6Al4V.

Table 2. Natural frequencies for the workpiece and tool.

\begin{tabular}{lll}
\hline & Direction & Natural frequency $(\mathrm{Hz})$ \\
\hline \multirow{3}{*}{ Workpiece } & $x$ & 1107 \\
& $y$ & 1220 \\
\multirow{2}{*}{ Tool } & $z$ & 1111,2945 \\
& $y$ & $1355,1900,2780,4912$ \\
\end{tabular}

Impact tests were conducted with impulse hammer and accelerometer PCB 352C65 to determine the frequency response functions (FRFs) of the machine tool-workpiece system (Fig. 4). The FRFs of the workpiece in the $x, y$ and $z$ directions were obtained near the location where the accelerometer was installed. As the hammer impact tests using the accelerometer are not easy to be conducted on the small tool tip in the $z$ direction, the FRF at the tool tip in this direction is not considered. Table 2 lists the dominant natural frequencies extracted from the FRFs. The workpiece has one dominant natural frequency (NF) in both the $x$ and $y$ directions, whereas two dominant NFs 1111 and $2945 \mathrm{~Hz}$ in the $z$ direction. For the tool, there are four dominant NFs around 1300, 1900, 2700 and $5000 \mathrm{~Hz}$ 
in the $x$ and $y$ directions, and the differences between the NFs in the two directions are small.
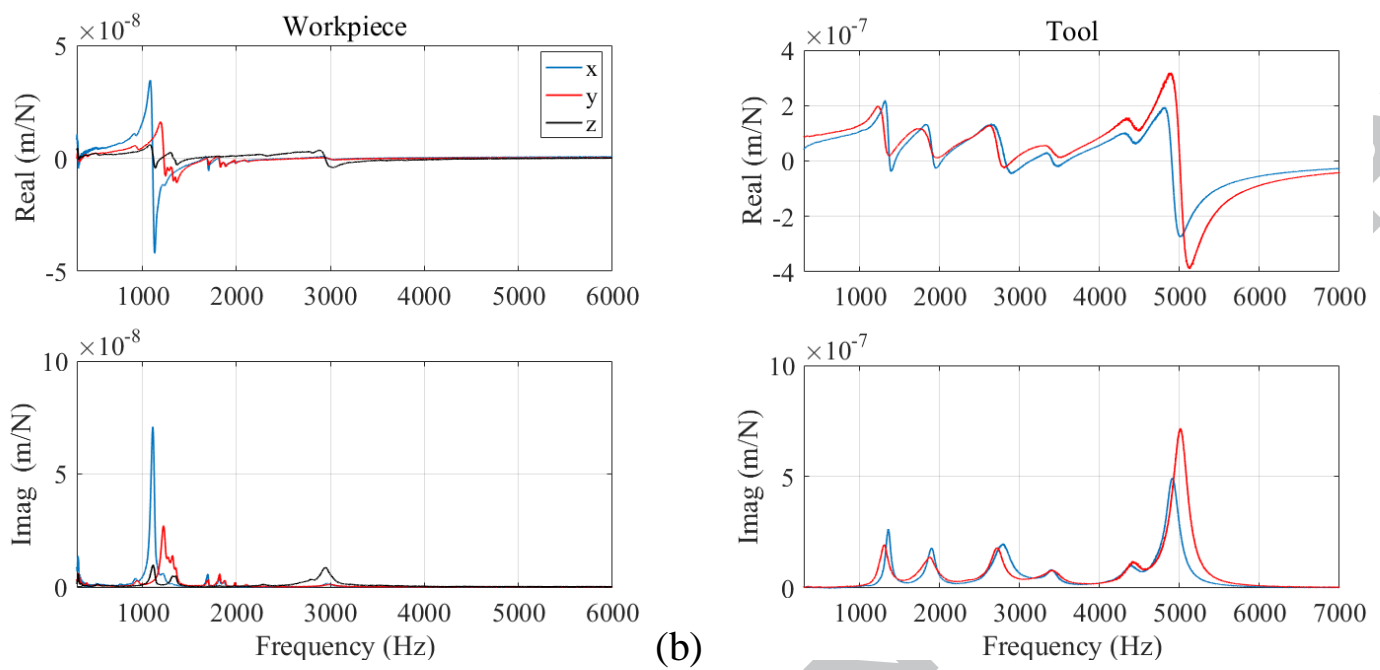

Fig. 4. Frequency response functions (a) workpiece (b) tool.

Table 3. Cutting parameters.

\begin{tabular}{l|l}
\hline Cutting parameters & Values \\
\hline Spindle speed $n_{p}(\mathrm{rpm})$ & $1100-7600$ with a 500 step \\
Feed per tooth $f_{t}(\mathrm{~mm} / \mathrm{tooth})$ & $0.08,0.1,0.12$ \\
Axial depth of cut $a_{p}(\mathrm{~mm})$ & $4,8,12$ \\
Radial depth of cut $a_{e}(\mathrm{~mm})$ & $0.5,1,1.5$ \\
\hline
\end{tabular}

The experimental tests were conducted under wide combinations of parameter settings.

The cutting conditions are given in Table 3 , and the total number of tests was 82 . As the radial depth of cut was low, all the tests were conducted under highly interrupted milling processing. Delio et al. [7] stated that the dynamometer was not suitable for interrupted machining processing. Furthermore, the most flexible NFs of the tool in the $x$ and $y$ directions were around $5000 \mathrm{~Hz}$ (Fig. 4), which were much larger than those around 3500 $\mathrm{Hz}$ of the dynamometer [30]. Therefore, the signals collected from the dynamometer are 
excluded, and only the vibration signals from the accelerometer are used for chatter detection.

\subsection{Empirical mode decomposition}

The signals from a stable and an unstable test are shown in Fig. 5. It can be seen that the stable test generates vibration signals with small amplitudes. However, the unstable test shows significant variations of the amplitudes, which indicates that the energy at chatter frequencies is not constant. The EEMD is used to process the signals of the two tests. The first four IMFs $c_{1}-c_{4}$ are given in Fig. 6, as IMFs $c_{5}$ and above are low frequency components about harmonics of the tooth passing frequency or spindle frequency. The amplitude of the white noise is selected to be $20 \%$ of the pre-processed signal, and the corresponding ensemble number $M=200$ is enough to control the residue noise in a reasonable range [23]. When the test becomes unstable, the amplitudes of IMFs $c_{1}-c_{4}$ increase, especially the amplitudes of IMFs $c_{1}$. The significant variations of IMFs $c_{1}$ indicate that $c_{1}$ in all the channels are the most informative frequency bands related to the machining states.
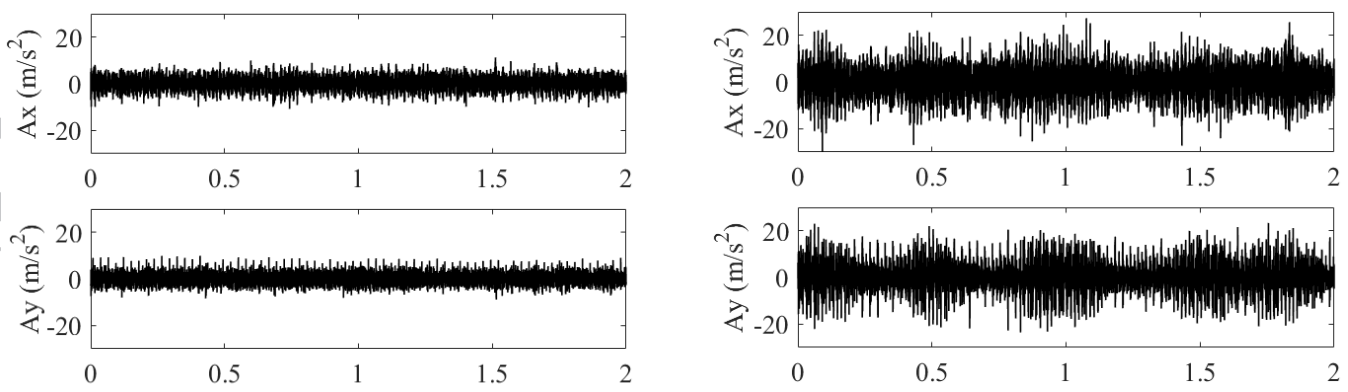

(a)
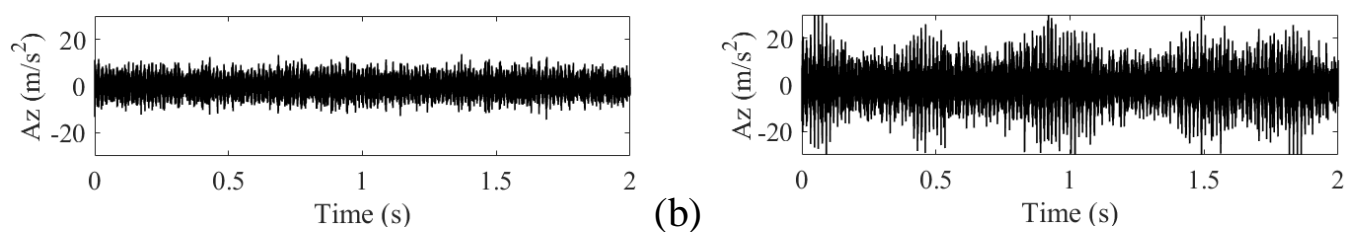

(b) 
Fig. 5. Vibration signals (a) stable $\left(n_{p}=2600 \mathrm{rpm}, f_{i}=0.1 \mathrm{~mm} /\right.$ tooth, $\left.a_{p}=12 \mathrm{~mm}, a_{e}=1 \mathrm{~mm}\right)$ (b) unstable $\left(n_{p}=2100 \mathrm{rpm}, f_{i}=0.12 \mathrm{~mm} /\right.$ tooth, $\left.a_{p}=12 \mathrm{~mm}, a_{e}=1 \mathrm{~mm}\right)$.
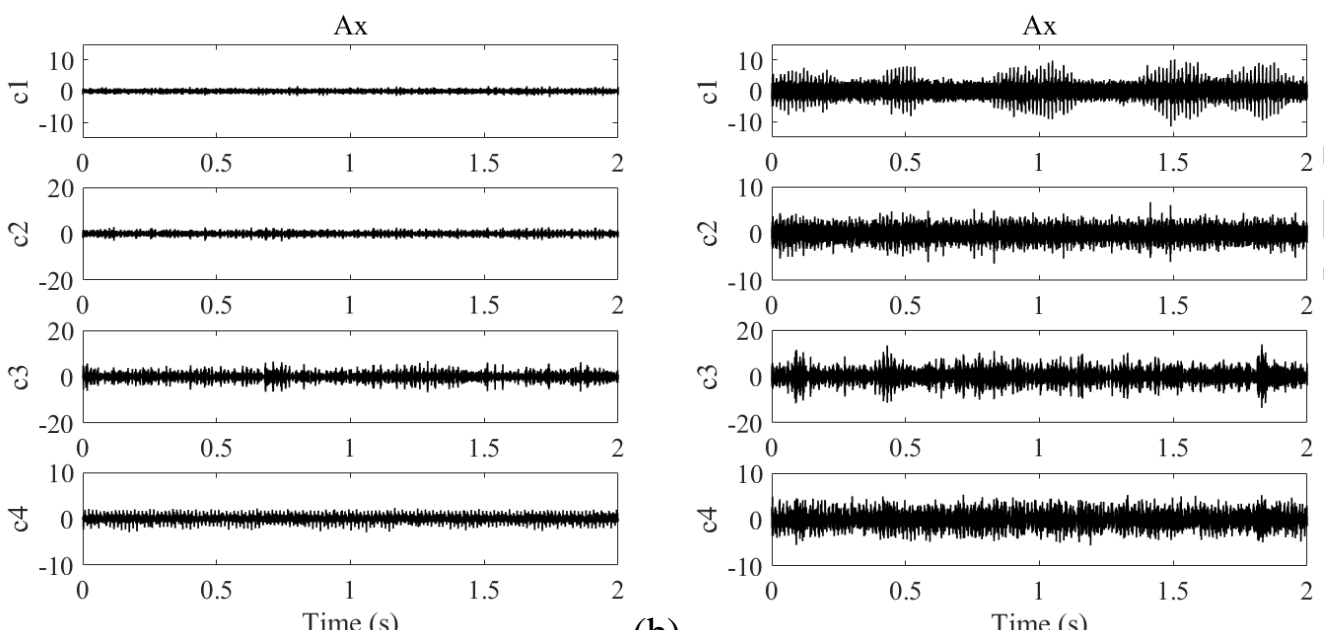

(a)

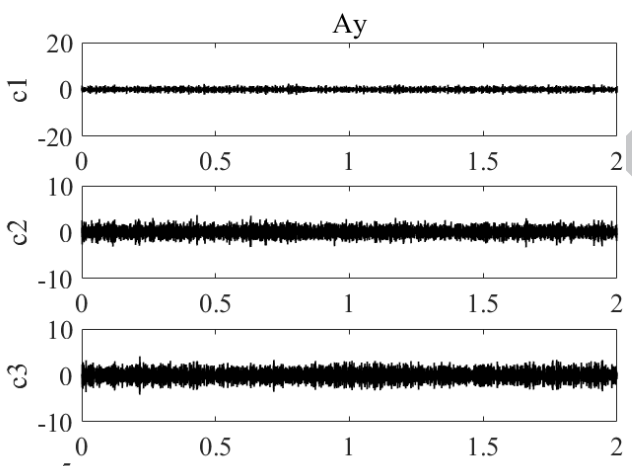

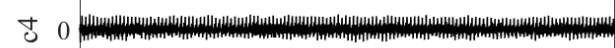

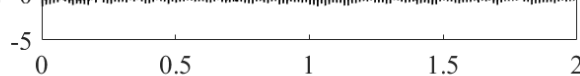

(c) Time (s) (b)
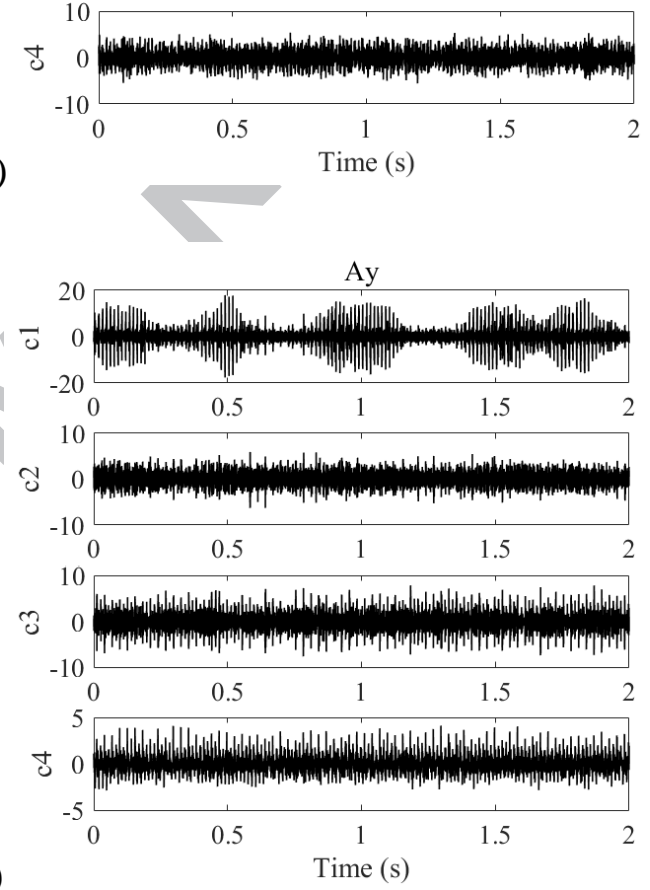
(e)
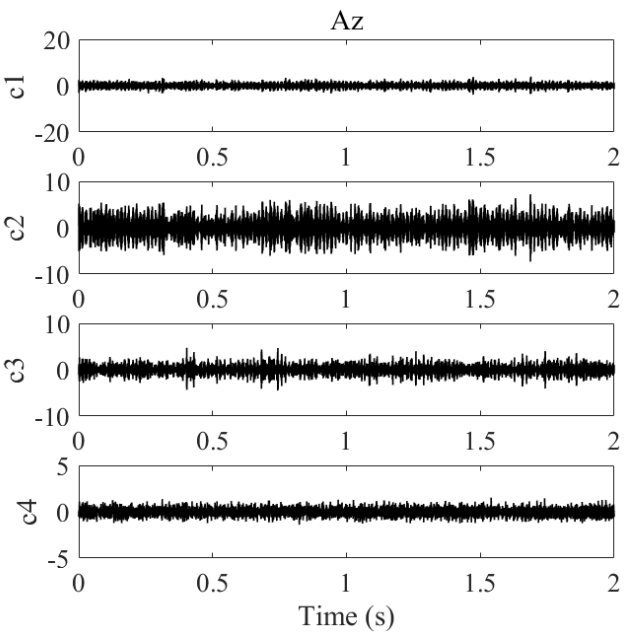
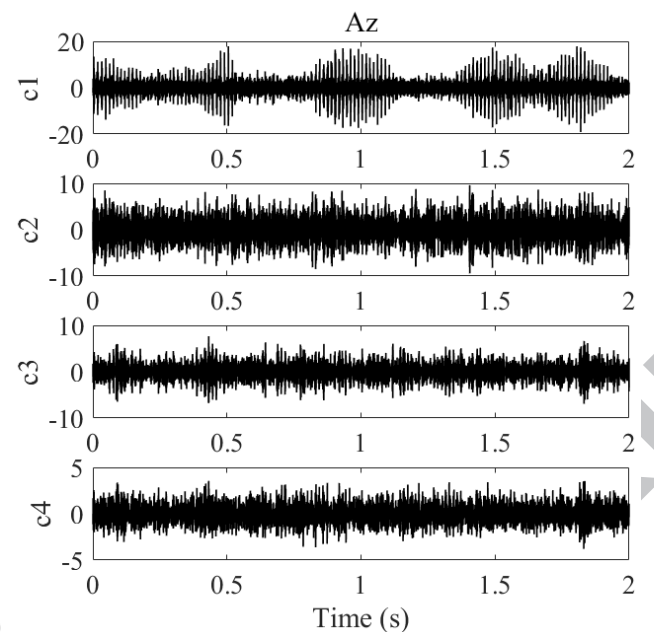

Fig. 6 IMFs of tests in Fig. 5. (a) $A x$, (c) $A y$ and (e) $A z$ for the stable test; (b) $A x$, (d) $A y$ and (f) $A z$ for the unstable test.

In order to further examine the properties of IMFs, the marginal spectra of IMFs from the Hilbert-Huang transform are presented in Fig. 7. It can be seen that each IMF represents a frequency band. IMF $c_{3}$ in each channel contains the NF around $1.1 \mathrm{kHz}$ of the workpiece and around $1.3 \mathrm{kHz}$ of the tool. The NFs around $2.7 \mathrm{kHz}$ of the tool and $2945 \mathrm{~Hz}$ of the workpiece in the $z$ direction are included in IMFs $c_{2}$. The marginal spectra of IMFs $c_{3}$ from the stable and unstable tests show significantly high peak values, especially in the $x$ and $z$ directions. This is due to the free vibration of the workpiece when the tool is not in cutting. The accelerometer that was attached to the fixture collected a vibration signal with a long free-vibration time in low immersion milling. Li and Liu [31] also considered that the workpiece/fixture was responsible for the large amplitudes around the workpiece NF. In the unstable test, the marginal spectra of IMFs $c_{1}$ significantly increase due to the energy rise around the most flexible NF (about $5 \mathrm{kHz}$ ) of the tool. The results evidence chatter occurrence with a frequency-dependent energy rise. The components that contain the 
feature frequencies (e.g. the most flexible NFs) are the informative frequency bands, and are usually decomposed from the raw signals to highlight the machining states.
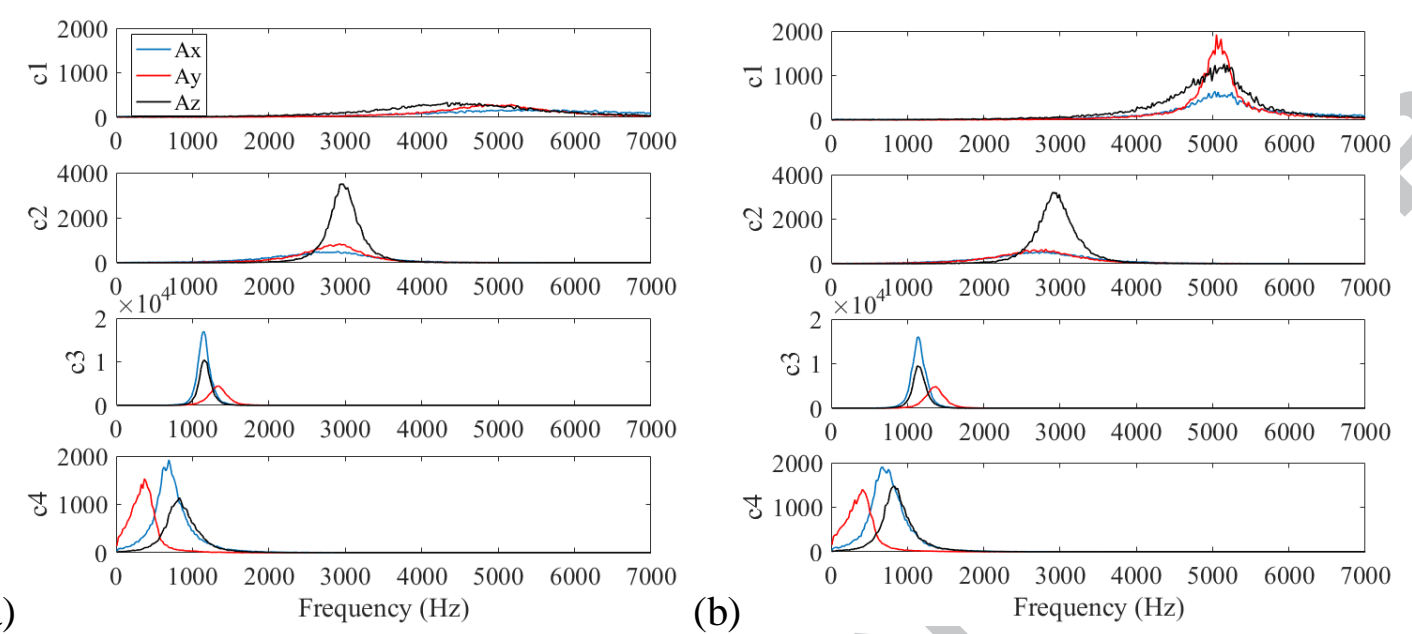

(a)

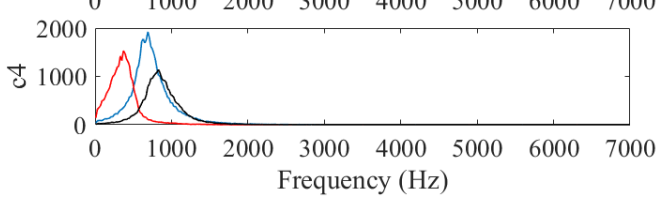

(b)

$$
\text { Frequency }(\mathrm{Hz})
$$

Fig. 7. Marginal spectra for tests in Fig. 5 (a) stable (b) unstable.

\subsection{Feature extraction and ranking}

The FDRs for features are given in Fig. 8. Except the crest factor $f_{15}$ and skewness $f_{16}$, the features from IMF $c_{1}$ in each channel have relatively large FDRs, indicating their superior separability capabilities. This implies again that IMF $c_{1}$ is the informative frequency band. Although the previous analysis results do not reveal that IMF $c_{3}$ is an important frequency band, it is interesting to observe that the energy ratios $f_{31}$ from IMFs $c_{3}$ have large FDRs, especially in the $A y$ and $A z$ channels. The top ranked features vary for different channels, as well as their FDRs. The standard variance of the $\mathrm{P} 2 \mathrm{P}$ per revolution $f_{13}$ is the top feature with a FDR of 0.96 in the $A x$ channel, and the energy ratio $f_{11}$ in the $A y$ channel has a FDR up to 2.27. Despite the variations of the feature ranking, the features $f_{11}, f_{12}, f_{13}, f_{14}, f_{17}$, and $f_{31}$ are the top six features in each channel. 

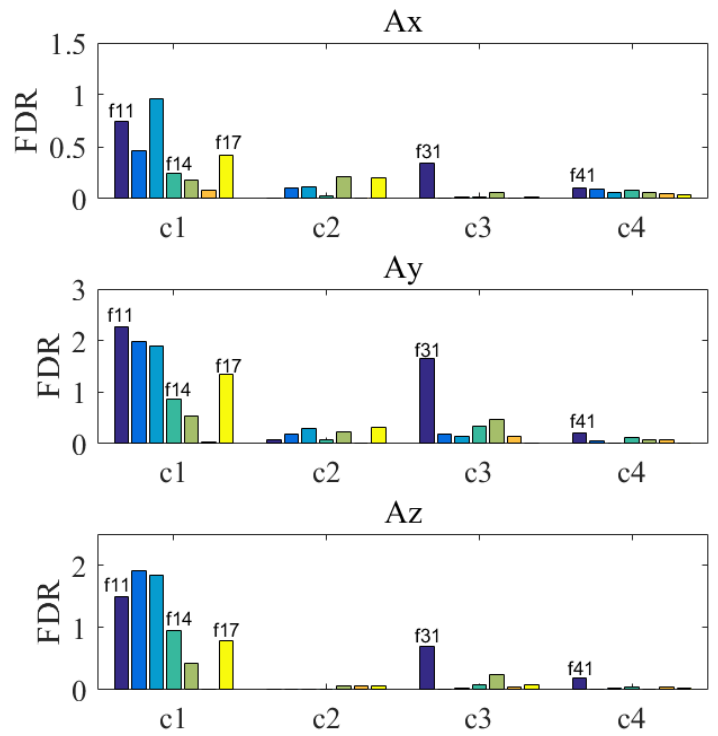

Fig. 8. Fishier discriminant ratios for features extracted from each IMF.

Three typical tests in Table 4 are selected for further examination of the top six features.

The machined surfaces and the vibration signals are shown in Fig. 9. The surface quality decreases from test 1 to test 3 , and the amplitudes of the vibration signals increase and become chaotic. Those observations indicate the severity of chatter increases from test 1 to test 3 . Fig. 10 shows the top six features for the three tests. The five features $f_{11}, f_{12}, f_{13}, f_{14}$, $f_{17}$ from IMF $c_{1}$ increase as the severity of chatter increases. However, the feature $f_{31}$ from $\mathrm{IMF} c_{3}$ decreases. This is because the energy rise of IMF $c_{1}$ with the increase in the severity of chatter makes the relative energy ratio $f_{31}$ decreases. It can be seen that the top six features can adequately describe the severity of chatter.

Table 4. Cutting conditions for three typical tests.

\begin{tabular}{l|llll}
\hline Test \# & $\begin{array}{l}n_{p} \\
\mathrm{rpm}\end{array}$ & $\begin{array}{l}f_{t} \\
\mathrm{~mm} / \text { tooth }\end{array}$ & $\begin{array}{l}a_{p} \\
\mathrm{~mm}\end{array}$ & $\begin{array}{l}a_{e} \\
\mathrm{~mm}\end{array}$ \\
\hline 1 & 2100 & 0.1 & 12 & 0.5 \\
2 & 1600 & 0.1 & 8 & 1.0 \\
3 & 2100 & 0.1 & 12 & 1.0 \\
\hline
\end{tabular}


(a)

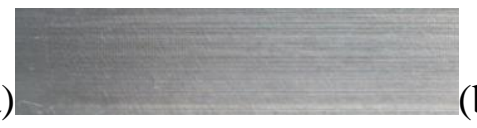

(b)
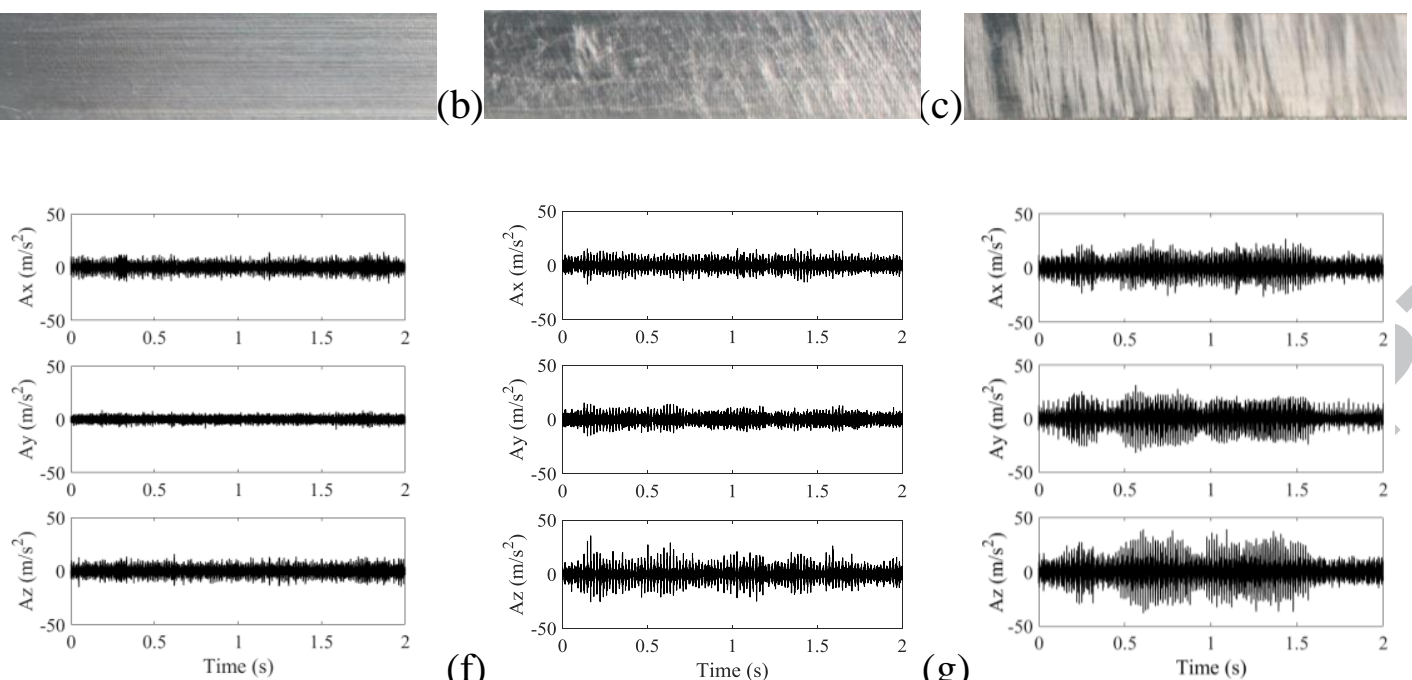

(e)

(f)

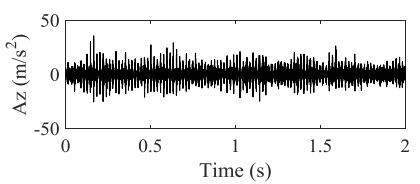

$(\mathrm{g})$
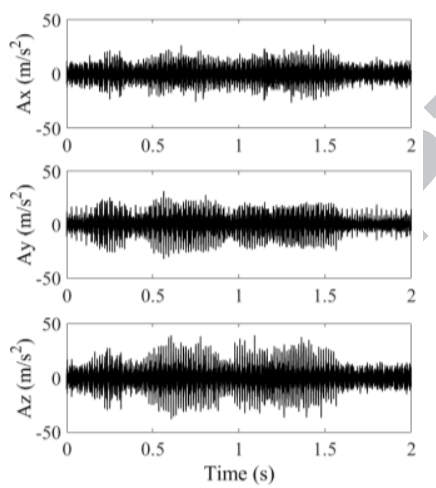

(20)

Fig. 9. Machined surfaces and vibration signals: (a) and (e) for stable test 1; (b) and (f) for unstable test 2; (c) and (g) for unstable test 3.
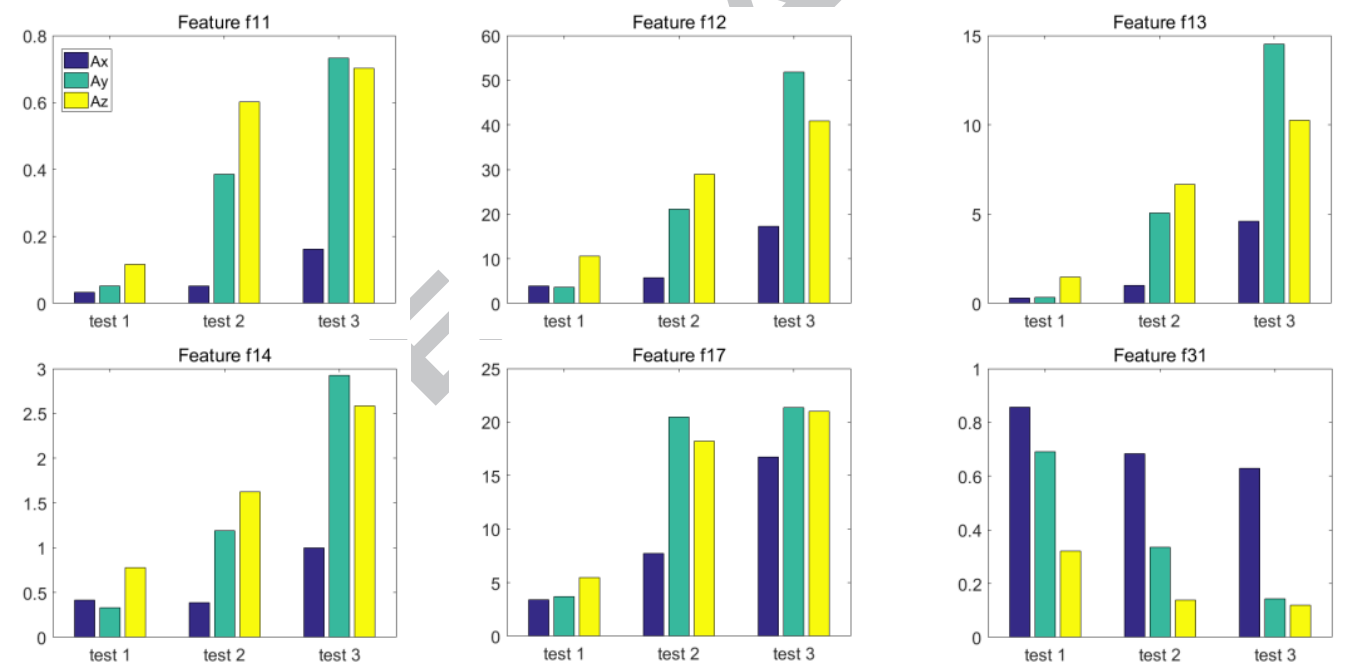

Fig. 10. Top six features for tests 1,2 and 3.

The stable and unstable tests in the two-dimensional space of the first and second ranked features are given in Fig. 11. Although the majority of the stable and unstable tests are well separated, the boundary between the stable and unstable tests is unclear. There exist continuous transients between stable and unstable machining states due to the complex machining system. In many studies, the machining processes are generally classified into 
three categories: stable, moderately unstable and unstable [16]. The moderately unstable states represent the transient or incipient states. It is not easy to identify distinct boundaries for moderately unstable processes due to the continuous transients of the system dynamics. Hence, this paper ignores the continuous transient states, and considers the moderately unstable tests as the unstable ones. On the other hand, the blurred boundary between the stable and unstable tests can also be caused by the fact that a space of two features has inadequate separability capability.
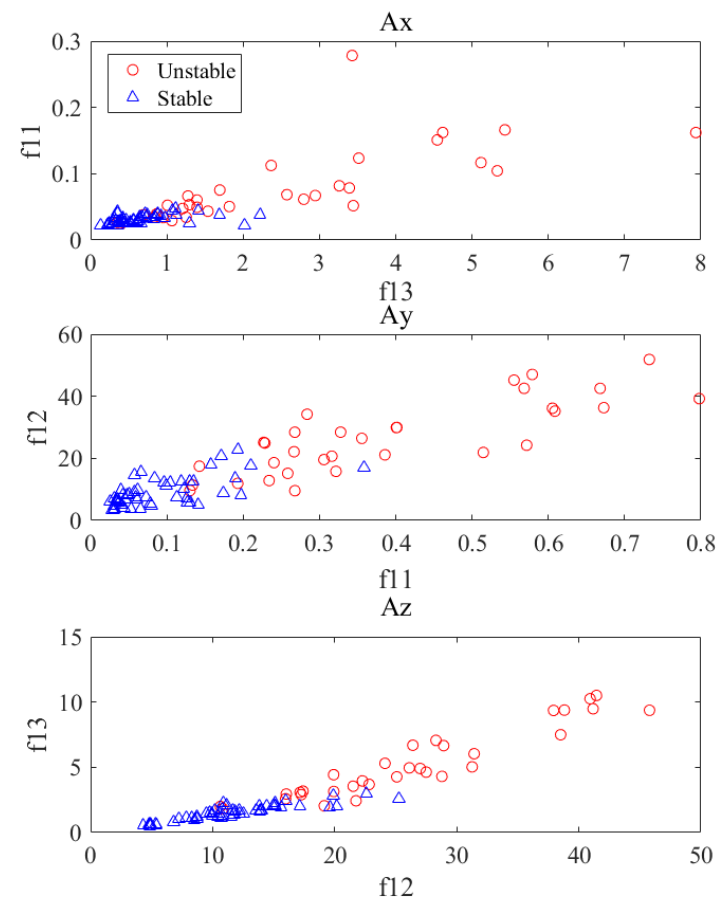

Fig. 11. Stable and unstable tests in the two-dimensional space of the first and second ranked features.

\subsection{Classification results}

According to the feature ranking results, the top six features are selected for each single-channel strategy. Three types of accuracies are defined for performance evaluation, namely, $A C_{\mathrm{sd}}, A C_{\mathrm{usd}}$ and $A C$, which are the percentages of stable tests classified as stable, unstable tests classified as unstable and total tests classified correctly, respectively. As the 
size of the experimental tests for chatter detection is small in this work, the leave-one-out (LOO) method [25] that is suitable for a limited data set is used to estimate the accuracies. The LOO method selects a different sample for testing, and the training is carried out using the excluded samples. This is repeated until all samples are tested. A linear SVM is applied for classification, as it gives better results than the nonlinear SVMs in this study. The accuracies for single-channel strategies are given in Fig. 12(a). The marginal spectra in Fig. 7 show that IMF $c_{1}$ of $A y$ reveals significant amplitude increases around $5 \mathrm{kHz}$ when the test is unstable. The top six features in the $A y$ channel have larger FDRs than those in other channels. For those reasons, $A y$ is the most effective channel with the highest classification accuracies among the three single-channel strategies. On the other hand, $A x$ shows the worst performance with an $A C_{\text {usd }}$ of only $71.9 \%$ in detection of the unstable tests.

The results from single-channel strategies are further presented to different multi-channel strategies. The accuracies estimated from the LOO method are shown in Fig. 12(a). All the investigated strategies give $A C_{\mathrm{sd}}$ higher than $A C_{\mathrm{usd}}$, which are also revealed in the work proposed by Kuljanic et al. [8]. This may be caused by the distributions of the stable and unstable tests in the space of the selected top six features. Fig. 11 reveals that the stable tests have a narrower distribution than the unstable tests, which is easier to be classified correctly. It is found that not all multi-channel strategies can improve the performance of chatter detection. The two-channel $(A x, A y)$ strategy shows the accuracies lower than the single-channel $A y$ strategy. On the other hand, the two fusion strategies $(A y, A z)$ and $(A x$, $A y, A z)$ present the best performance with the same accuracies, $A C_{\text {sd }} 96.0 \%, A C_{\text {usd }} 93.0 \%$ and $A C 95.1 \%$. It is noted that these two fusion strategies significantly improve the ability 
of the monitoring system to identify unstable tests, as $A C_{\mathrm{usd}} 93.0 \%$ for the two strategies is larger than $A C_{\text {usd }} 84.4 \%$ for the single-channel $A y / A z$ strategy and the two-channel $(A x, A y)$ strategy. For an online monitoring system with a high demand of computation time saving, the two-channel $(A y, A z)$ strategy with a less size of features is recommended.

(a)

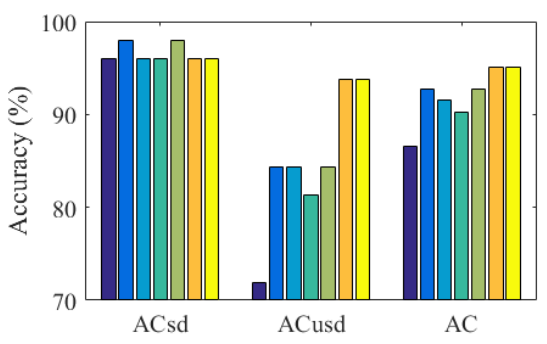

(c)

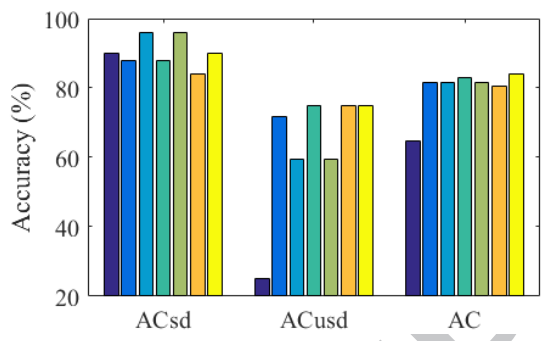

(b)
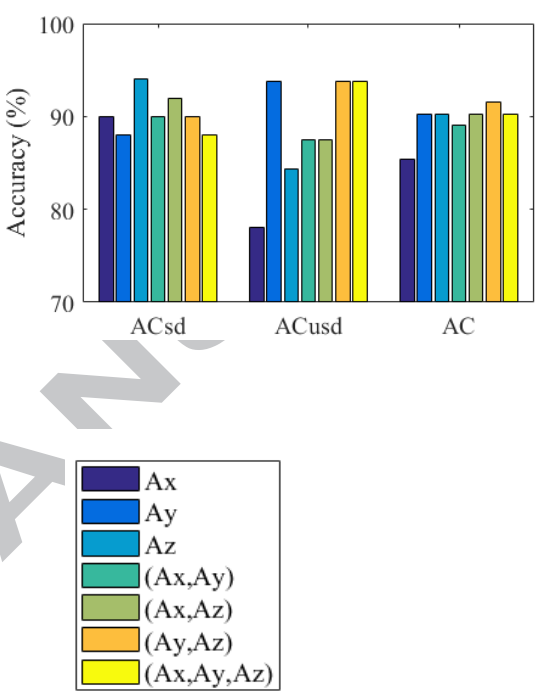

Fig. 12. Classification accuracies (a) the proposed approach (b) the first approach (c) the second approach.

\subsection{Discussions}

For further evaluation, the proposed approach is compared with two additional approaches:

the first approach inputs all features into the classifier without feature ranking/selection;

the second approach generates the features from the raw signals without EEMD. The comparison aims to reveal the importance of feature ranking/selection and signal processing procedures. 

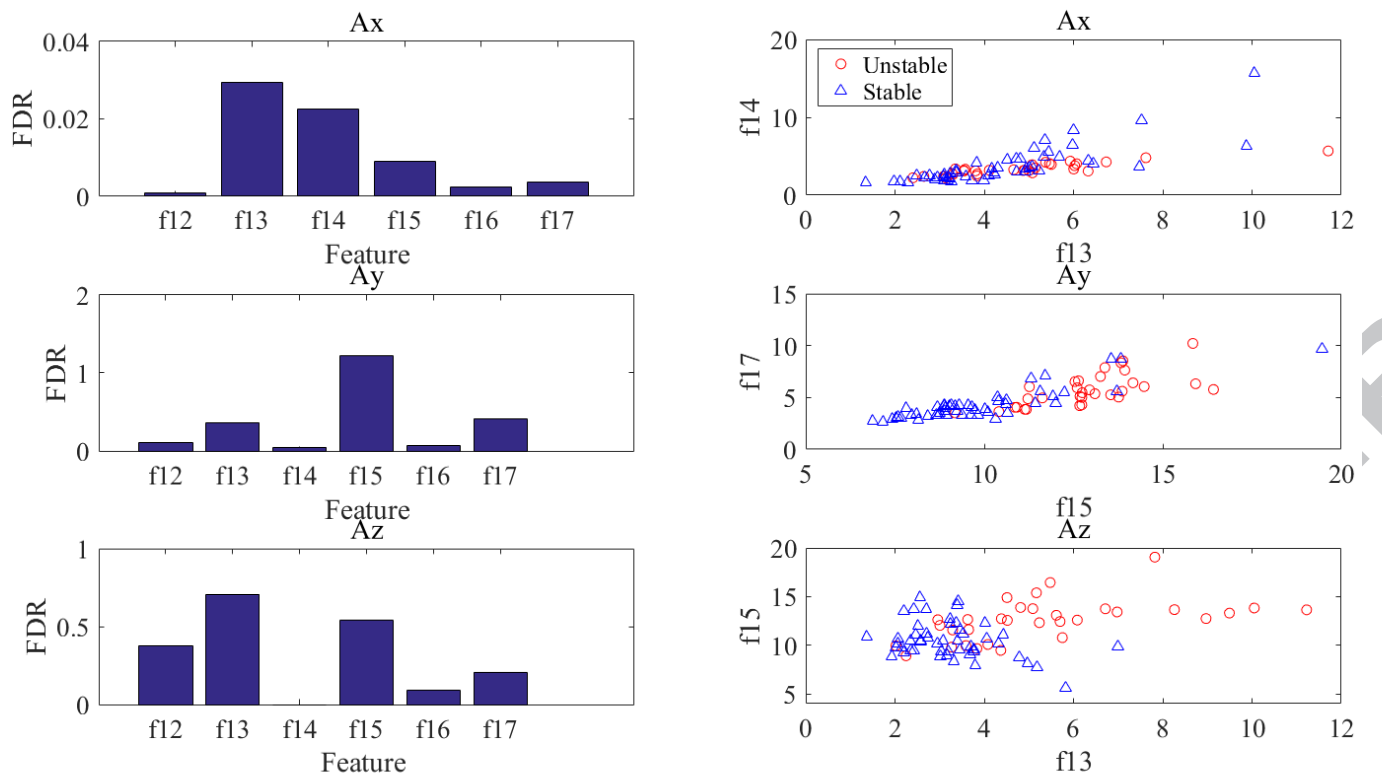

(a)

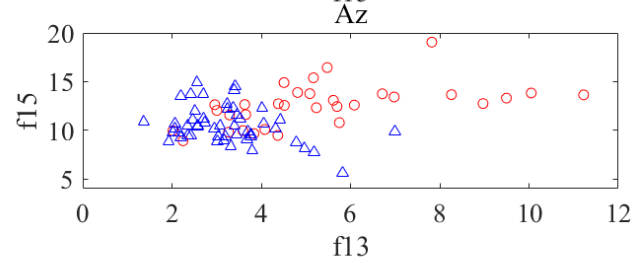

(b)

Fig. 13. Analysis results for the second approach (a) FDRs of all features (b) stable and unstable results in the space of the top two features.

The results estimated from the first approach are given in Fig. 12(b). The two-channel ( $A y$, $A z$ ) strategy gives the highest $A C_{\text {usd }}$ and $A C, 93.8 \%$ and $91.5 \%$, respectively. The first approach has lower $A C_{\mathrm{sd}}$ in classifying stable tests than the proposed one, resulting in the decrease of the $A C$ s from all the investigated strategies. For the second approach, FDRs of features are shown in Fig. 13(a). The energy ratio $f_{11}$ is ignored in this approach, as the features are extracted from the raw signals without decomposition. FDRs are much smaller than those from the proposed approach. Fig. 13(b) reveals that the stable and unstable tests cannot be separated adequately by the top two features, especially in the Ax channel. Fig. 12(c) gives the classification results based on the top three features in each channel. The single-channel $A x$ strategy in this approach has an accuracy of only $25.0 \%$ in classifying the unstable tests. The second approach presents the lowest $A C$ s in classifying the total tests, indicating the significance of the decomposition of the raw signals into frequency bands. 
The computation time that the proposed approach and the two additional approaches take for classification is listed in Table 5. In each approach, all the seven strategies (i.e. single-channel or multi-channel strategies) are examined. Except the single-channel $A y$ strategy and the two-channel $(A x, A z)$ strategy, the computation time in the first additional approach is longer than its corresponding one in the proposed approach for the remain five strategies. All the strategies in the second approach take the shortest time among the three approaches. However, the performance of the best strategy $(A x, A y, A z)$ in the second approach is lower than that of the best strategies in the other two approaches. The best strategy $(A y, A z)$ in the proposed approach gives not only the highest $A C_{\text {sd }}, A C_{\text {usd }}$ and $A C$, but also shorter computation time than the best strategy $(A y, A z)$ in the first approach. Hence, the two-channel $(A y, A z)$ strategy in the proposed approach is recommended.

Table 5. Comparison of Computation time for each approach

\begin{tabular}{l|lll}
\hline \multirow{2}{*}{ Strategies } & \multicolumn{3}{|l}{ Computation time (s) } \\
\cline { 2 - 4 } & Proposed approach & First approach & Second approach \\
\hline$A x$ & 1.16 & 1.71 & 0.95 \\
$A y$ & 1.50 & 1.10 & 0.98 \\
$A z$ & 1.13 & 1.21 & 1.05 \\
$(A x, A y)$ & 3.52 & 3.80 & 2.93 \\
$(A x, A z)$ & 3.28 & 3.23 & 2.89 \\
$(A y, A z)$ & 3.37 & 3.60 & 3.28 \\
$(A x, A y, A z)$ & 4.47 & 4.90 & 4.12 \\
\hline
\end{tabular}

\section{Conclusions}

In this paper, an intelligent chatter detection method based on EEMD and feature selection is proposed for a multi-channel monitoring system. As chatter is coupled with energy rises around natural frequencies of the machining system, EEMD is therefore used to decompose the raw signals into a set of IMFs covering single or multiple natural 
frequencies. The FDR that quantifies the separability capabilities of individual features is used to identify the informative features from IMFs. A linear SVM is used for classification, and its performance is evaluated using the LOO method.

The chatter detection method is implemented in low immersion milling of titanium alloy Ti6Al4V under various cutting conditions. The most significant energy rise around the most flexible natural frequency of the tool is identified in the $A y$ channel when chatter occurs. The Ay channel is the most effective to detect chatter when a single-channel strategy is used. On the other hand, the fusion strategy based on either the channels $(A y, A z)$ or $(A x, A y, A z)$ presents the best performance in classifying the stable and unstable tests. The two-channel $(A y, A z)$ strategy with the smaller size of features and shorter computation time is preferred for future development of an online monitoring system. Two additional approaches that ignore either feature selection or EEMD are compared with the proposed one. The results show that the additional approaches give much lower accuracies than the proposed one, indicating the importance of feature ranking/selection and signal processing procedures.

\section{Acknowledgements}

This study was partially supported by the Collaborative Innovation Center of High-End Equipment Manufacturing in Fujian. The authors would like to express their acknowledgements to the Advanced Manufacturing Laboratory, UNSW, for the support of the experimental work. Comments and suggestions from reviewers are greatly appreciated. 


\section{References}

[1] G. Quintana, J. Ciurana, Chatter in machining processes: A review, International Journal of Machine Tools and Manufacture, 51 (2011) 363-376.

[2] W.X. Tang, Q.H. Song, S.Q. Yu, S.S. Sun, B.B. Li, B. Du, X. Ai, Prediction of chatter stability in high-speed finishing end milling considering multi-mode dynamics, Journal of Materials Processing Technology, 209 (2009) 2585-2591.

[3] S. Tangjitsitcharoen, In-process monitoring and detection of chip formation and chatter for CNC turning, Journal of Materials Processing Technology, 209 (2009) 4682-4688.

[4] H. Cao, Y. Lei, Z. He, Chatter identification in end milling process using wavelet packets and Hilbert-Huang transform, International Journal of Machine Tools and Manufacture, 69 (2013) 11-19.

[5] J. Griffin, X. Chen, Multiple classification of the acoustic emission signals extracted during burn and chatter anomalies using genetic programming, Int J Adv Manuf Technol, 45 (2009) 1152-1168.

[6] T. Segreto, A. Simeone, R. Teti, Multiple Sensor Monitoring in Nickel Alloy Turning for Tool Wear Assessment via Sensor Fusion, Procedia CIRP, 12 (2013) 85-90.

[7] T. Delio, J. Tlusty, S. Smith, Use of audio signals for chatter detection and control, Journal of Engineering for Industry, 114 (1992) 146-157.

[8] E. Kuljanic, G. Totis, M. Sortino, Development of an intelligent multisensor chatter detection system in milling, Mechanical Systems and Signal Processing, 23 (2009) 1704-1718.

[9] E. Kuljanic, M. Sortino, G. Totis, Multisensor approaches for chatter detection in milling, Journal of Sound and Vibration, 312 (2008) 672-693.

[10] T.L. Schmitz, K. Medicus, B. Dutterer, Exploring once-per-revolution audio signal variance as a chatter indicator, Machining Science and Technology, 6 (2002) 215-233.

[11] L. Vela-Martínez, J.C. Jáuregui-Correa, J. Álvarez-Ramírez, Characterization of machining chattering dynamics: An R/S scaling analysis approach, International Journal of Machine Tools and Manufacture, 49 (2009) 832-842.

[12] M.A.U. Patwari, A.K.M.N. Amin, W.F. Faris, Influence of chip serration frequency on chatter formation during end milling of Ti6A14V, Journal of Manufacturing Science and Engineering, Transactions of the ASME, 133 (2011). 
[13] H. Li, X. Jing, J. Wang, Detection and analysis of chatter occurrence in micro-milling process, Proceedings of the Institution of Mechanical Engineers, Part B: Journal of Engineering Manufacture, 228 (2014) 1359-1371.

[14] E. Al-Regib, J. Ni, Chatter detection in machining using nonlinear energy operator, Journal of Dynamic Systems, Measurement, and Control, 132 (2010) 034502-034502.

[15] M. Lamraoui, M. Barakat, M. Thomas, M.E. Badaoui, Chatter detection in milling machines by neural network classification and feature selection, Journal of Vibration and Control, 21 (2013) 1251-1266.

[16] Z. Yao, D. Mei, Z. Chen, On-line chatter detection and identification based on wavelet and support vector machine, Journal of Materials Processing Technology, 210 (2010) 713-719.

[17] R. Teti, K. Jemielniak, G. O’Donnell, D. Dornfeld, Advanced monitoring of machining operations, CIRP Annals - Manufacturing Technology, 59 (2010) 717-739.

[18] J. Dong, K.V.R. Subrahmanyam, Y.S. Wong, G.S. Hong, A.R. Mohanty, Bayesian-inference-based neural networks for tool wear estimation, The International Journal of Advanced Manufacturing Technology, 30 (2006) 797-807.

[19] S. Theodoridis, K. Koutroumbas, Chapter 6 - Feature Generation I: Data Transformation and Dimensionality Reduction, Pattern Recognition (Fourth Edition), Academic Press, Boston, 2009, pp. 323-409.

[20] M. Siddhpura, R. Paurobally, A review of chatter vibration research in turning, International Journal of Machine Tools and Manufacture, 61 (2012) 27-47.

[21] Y. Fu, Y. Zhang, H. Zhou, D. Li, H. Liu, H. Qiao, X. Wang, Timely online chatter detection in end milling process, Mechanical Systems and Signal Processing, 75 (2016) 668-688.

[22] Y. Peng, Empirical Model Decomposition Based Time-Frequency Analysis for the Effective Detection of Tool Breakage, Journal of Manufacturing Science and Engineering, 128 (2004) 154-166.

[23] N.E. Huang, Z. Wu, Ensemble empirical mode decomposition: a noise-assisted data analysis method, Advances in Adaptive Data Analysis, 01 (2009) 1-41. 
[24] J.R. Yeh, J.S. Shieh, N.E. Huang, Complementary ensemble empirical mode decomposition: a novel noise enhanced data analysis method, Advances in Adaptive Data Analysis, 02 (2010) 135-156.

[25] S. Theodoridis, K. Koutroumbas, Chapter 5 - Feature Selection, Pattern Recognition (Fourth Edition), Academic Press, Boston, 2009, pp. 261-322.

[26] C.J.C. Burges, A Tutorial on Support Vector Machines for Pattern Recognition, Data Mining and Knowledge Discovery, 2 (1998) 121-167.

[27] S. Theodoridis, K. Koutroumbas, Chapter 3 - Linear Classifiers, Pattern Recognition (Fourth Edition), Academic Press, Boston, 2009, pp. 91-150.

[28] S. Theodoridis, K. Koutroumbas, Chapter 4 - Nonlinear Classifiers, Pattern Recognition (Fourth Edition), Academic Press, Boston, 2009, pp. 151-260.

[29] B. Waske, J.A. Benediktsson, Fusion of Support Vector Machines for Classification of Multisensor Data, IEEE Transactions on Geoscience and Remote Sensing, 45 (2007) 3858-3866.

[30] Kistler, Multicomponent Dynamometer Type 9257, 2009.

[31] Y. Li, Q. Liu, Chatter identification in CNC milling based on wallet packet and Hilbert-Huang transform, Computer Integrated Manufacturing System, 21 (2015) 204-216. 
- A new chatter detection method using EEMD and feature selection is presented

- EEMD highlights chatter characteristics in narrow decomposed frequency bands

- FDR-based feature selection improves classification speed and performance

- Fusion of two specific channel vibration signals gives better detection accuracy 


\section{Accepted Manuscript}

An Intelligent Chatter Detection Method Based on EEMD and Feature Selection with Multi-channel Vibration Signals

Yun Chen, Huaizhong Li, Liang Hou, Jun Wang, Xiangjian Bu

PII:

S0263-2241(18)30532-3

DOI: https://doi.org/10.1016/j.measurement.2018.06.006

Reference: MEASUR 5623

To appear in:

Measurement

Received Date: $\quad 11$ August 2017

Revised Date: $\quad 7$ May 2018

Accepted Date: $\quad 5$ June 2018

Please cite this article as: Y. Chen, H. Li, L. Hou, J. Wang, X. Bu, An Intelligent Chatter Detection Method Based on EEMD and Feature Selection with Multi-channel Vibration Signals, Measurement (2018), doi: https://doi.org/ 10.1016/j.measurement.2018.06.006

This is a PDF file of an unedited manuscript that has been accepted for publication. As a service to our customers we are providing this early version of the manuscript. The manuscript will undergo copyediting, typesetting, and review of the resulting proof before it is published in its final form. Please note that during the production process errors may be discovered which could affect the content, and all legal disclaimers that apply to the journal pertain. 